\title{
HIF-1a and TAZ serve as reciprocal co-activators in human breast cancer cells
}

\author{
Lisha Xiang 1,2,3, Daniele M. Gilkes 2,3, Hongxia Hu2,3, Weibo Luo ${ }^{2,3}$, John W. Bullen ${ }^{2,3}$, \\ Houjie Liang ${ }^{1}$ and Gregg L. Semenza ${ }^{2,3,4}$ \\ ${ }^{1}$ Department of Oncology and Southwest Cancer Center, Southwest Hospital, Third Military Medical University, Chongqing, \\ China \\ ${ }^{2}$ Vascular Program, Institute for Cell Engineering, Johns Hopkins University School of Medicine, Baltimore, MD, USA \\ ${ }^{3}$ McKusick-Nathans Institute of Genetic Medicine, Johns Hopkins University School of Medicine, Baltimore, MD, USA \\ ${ }^{4}$ Departments of Pediatrics, Medicine, Oncology, Radiation Oncology, and Biological Chemistry, Johns Hopkins University \\ School of Medicine, Baltimore, MD, USA \\ Correspondence to: Gregg L. Semenza, email: gsemenza@jhmi.edu \\ Keywords: breast cancer progression, hypoxia-inducible factor 1, MDA-MB-231 cells, MCF-7 cells \\ Received: May 05, $2015 \quad$ Accepted: May 05, $2015 \quad$ Published: May 19, 2015
}

This is an open-access article distributed under the terms of the Creative Commons Attribution License, which permits unrestricted use, distribution, and reproduction in any medium, provided the original author and source are credited.

\section{ABSTRACT}

Hypoxia-inducible factor 1a (HIF-1a) expression is a hallmark of intratumoral hypoxia that is associated with breast cancer metastasis and patient mortality. Previously, we demonstrated that HIF-1 stimulates the expression and activity of $T A Z$, which is a transcriptional effector of the Hippo signaling pathway, by increasing TAZ synthesis and nuclear localization. Here, we report that direct protein-protein interaction between HIF-1a and TAZ has reciprocal effects: HIF-1a stimulates transactivation mediated by TAZ and TAZ stimulates transactivation mediated by HIF1a. Inhibition of TAZ expression impairs the hypoxic induction of HIF-1 target genes, such as PDK1, LDHA, BNIP3 and P4HA2 in response to hypoxia, whereas inhibition of HIF-1a expression impairs TAZ-mediated transactivation of the CTGF promoter. Taken together, these results complement our previous findings and establish bidirectional crosstalk between HIF-1a and TAZ that increases their transcriptional activities in hypoxic cells.

\section{INTRODUCTION}

Intratumoral hypoxia is a critical microenvironmental factor driving breast cancer progression $[1,2]$. In response to reduced $\mathrm{O}_{2}$ availability, hypoxia-inducible factor 1 (HIF-1) mediates the transcriptional activation of genes encoding proteins that are required for many important steps in breast cancer progression, such as angiogenesis, cancer stem cell maintenance, cell motility, epithelial-mesenchymal transition, extracellular matrix remodeling, metabolic reprogramming, metastasis, and resistance to therapy [3-6]. HIF-1 is a heterodimeric transcription factor, which consists of two subunits, HIF- $1 \alpha$ and HIF$1 \beta$ [7]. In normoxic cells, HIF-1 $\alpha$ is hydroxylated by prolyl hydroxylases (PHD1-3) at proline residue 402 and/or 564, and by an asparaginyl hydroxylase (FIH1) at asparagine residue 803 [reviewed in ref. 8]. The von Hippel-Lindau tumor suppressor protein binds to prolyl hydroxylated HIF-1 $\alpha$ and recruits an E3 ubiquitin ligase complex containing Elongin $\mathrm{C}$, Elongin B, and Cullin 2 that ubiquitinates HIF-1 $\alpha$ and thereby targets it for degradation by the $26 S$ proteasome. In addition, asparaginyl hydroxylation of HIF- $1 \alpha$ blocks its binding to the transcriptional co-activator p300 and reduces HIF1 transcriptional activity. Under hypoxic conditions, prolyl and asparaginyl hydroxylation are inhibited, which stabilizes the HIF-1 $\alpha$ protein and promotes HIF-1dependent gene transcription [reviewed in ref. 8].

A growing number of proteins have been identified that function as co-activators of HIF-1 by binding to HIF$1 \alpha$ and regulating its transcriptional activity. The histone acetyltransferase p300 catalyzes the acetylation of lysine residues on the N-terminal tail of core histones at HIF-1 target genes, leading to changes in chromatin structure that promote HIF-1-dependent gene transcription [9]. Pyruvate 
kinase M2 (PKM2) also interacts with HIF-1 $\alpha$ in the cell nucleus and functions as a transcriptional co-activator in HeLa cervical carcinoma and Hep3B hepatoblastoma cells [10]. PKM2 increased HIF-1 binding to hypoxia response elements (HREs) at target genes, recruitment of p300, histone acetylation, and subsequent transactivation of HIF-1 target genes including SLC2A1, LDHA, and PDK1, which encode glucose transporter 1 , lactate dehydrogenase A, and pyruvate dehydrogenase kinase 1 , respectively [10]. HIF-1 $\alpha$ also recruits histone demethylases including JMJD2C, which demethylates trimethylated lysine 9 of histone $\mathrm{H} 3$ and enhances HIF-1 binding to HREs to activate transcription of HIF-1 target genes and drive breast cancer metastasis [11]. Other co-activators of HIF1 that promote breast cancer progression include Pontin [12] and XBP1s [13].

We reported recently that HIF-1, but not HIF2, binds directly to the WWTR1 gene encoding the transcriptional co-activator with PDZ-binding motif (TAZ), an effector of Hippo signaling [5]. TAZ interacts with DNA binding proteins of the TEA/ATTS domain (TEAD) family to activate transcription of target genes, such as $C T G F$ (encoding connective tissue growth factor), which promote epithelial-mesenchymal transition and the breast cancer stem-cell phenotype [14-18]. In addition to increasing TAZ mRNA and protein expression, HIF1 transactivates the SIAH1 gene, which encodes an ubiquitin protein ligase that is required for the proteasomal degradation of LATS2, a kinase that phosphorylates TAZ and inhibits its nuclear localization [5]. Thus, HIF-1 coordinately regulates TAZ expression and TAZ/TEAD transcriptional activity. HIF-1-dependent TAZ activity induces the breast cancer stem cell phenotype in response to hypoxia [5].

TAZ was reported to interact with HIF-1 $\alpha$ and positively regulate HIF-1 transcriptional activity in a breast cancer cell line that was selected for metastasis to bone [18]. Here, we report that TAZ and HIF-1 $\alpha$ interact in MDA-MB-231 cells, which metastasize to lymph nodes and lungs in a HIF-dependent manner [19, 20]. Remarkably, we also report that the interaction of HIF$1 \alpha$ with TAZ also stimulates TAZ/TEAD transcriptional activity. Thus, bidirectional functional interactions between HIF-1 $\alpha$ and TAZ synergistically drive the expression of downstream target genes in hypoxic breast cancer cells.

\section{RESULTS}

\section{TAZ serves as a co-activator for HIF-1a}

We previously established MDA-MB-231 subclones, which were stably transduced with a lentiviral expression vector encoding a non-targeting control (NTC) short hairpin RNA (shRNA) or either of two independent shRNAs targeting TAZ (shT1 and shT2), and immunoblot assays confirmed effective knockdown of TAZ protein expression, with no effect on HIF-1 $\alpha$ protein levels [5]. To determine whether TAZ regulates HIF-1 transcriptional activity, MDA-MB-231 subclones were co-transfected with HIF-1-dependent reporter plasmid p2.1, which contains an HRE from the human ENO1 gene upstream of SV40 promoter and firefly luciferase coding sequences [21], and control reporter pSV-Renilla, which contains the SV40 promoter upstream of Renilla luciferase coding sequences. The ratio of firefly:Renilla luciferase activity is a measure of HIF-1 transcriptional activity. Knockdown of endogenous TAZ expression significantly reduced HIF-1 transcriptional activity under hypoxic conditions (Figure 1A).

To determine whether TAZ directly regulates HIF$1 \alpha$ transactivation domain (TAD) function, MDA-MB-231 NTC and TAZ-knockdown subclones were co-transfected with the following plasmids: expression vector pGalA [22], which encodes the GAL4 DNA-binding domain fused to the HIF-1 $\alpha$ TAD (amino acid residues 531-826) or pGalO, which encodes only the GAL4 DNA-binding domain; reporter plasmid pG5E1bLuc, which contains five GAL4-binding sites and a TATA box from the adenovirus E1b gene upstream of firefly luciferase coding sequences; and pSV-Renilla. Exposure of the cells to $1 \% \mathrm{O}_{2}$ for 24 $\mathrm{h}$ increased GalA-dependent, but not GalO-dependent, luciferase activity in MDA-MB-231 cells transfected with NTC vector, whereas GalA-dependent transcription was significantly decreased in cells transfected with vector encoding TAZ shRNA (Figure 1B).

To determine whether TAZ stimulates HIF-1 $\alpha$ TAD function by direct interaction, glutathione- $S$-transferase (GST) and a recombinant GST-HIF-1 $\alpha$ (531-826) fusion protein were purified from bacteria, incubated with whole cell lysates and glutathione-agarose beads, and the bound proteins were analyzed by immunoblot assays using antiTAZ and anti-GST antibodies, which revealed binding of TAZ to GST-HIF-1 $\alpha(531-826)$ but not to GST alone (Figure 1C). Interaction of endogenous TAZ and HIF-1 $\alpha$ in hypoxic MDA-MB-231 cells was also demonstrated by co-immunoprecipitation assays, using anti-HIF$1 \alpha$ antibody to precipitate TAZ (Figure 1D) and antiTAZ antibody to precipitate HIF- $1 \alpha$ (Figure 1E). Taken together, these results demonstrate that TAZ functions as a co-activator for HIF-1-dependent gene transcription.

\section{HIF-1 $\alpha$ serves as a co-activator for TAZ}

We next investigated whether HIF-1 $\alpha$ regulates TAZ transcriptional activity. We established MCF-7 breast cancer subclones, which were stably transduced with lentiviral vectors encoding NTC shRNA or shRNA targeting HIF-1 $\alpha(\operatorname{sh} 1 \alpha)$, HIF-1 $\beta(\operatorname{sh} 1 \beta)$, or TAZ (shT1), and immunoblot assays confirmed effective knockdown of 
A

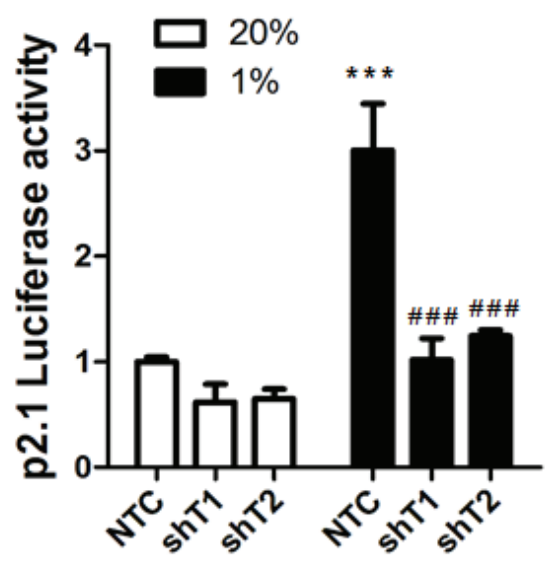

$\mathrm{B}$

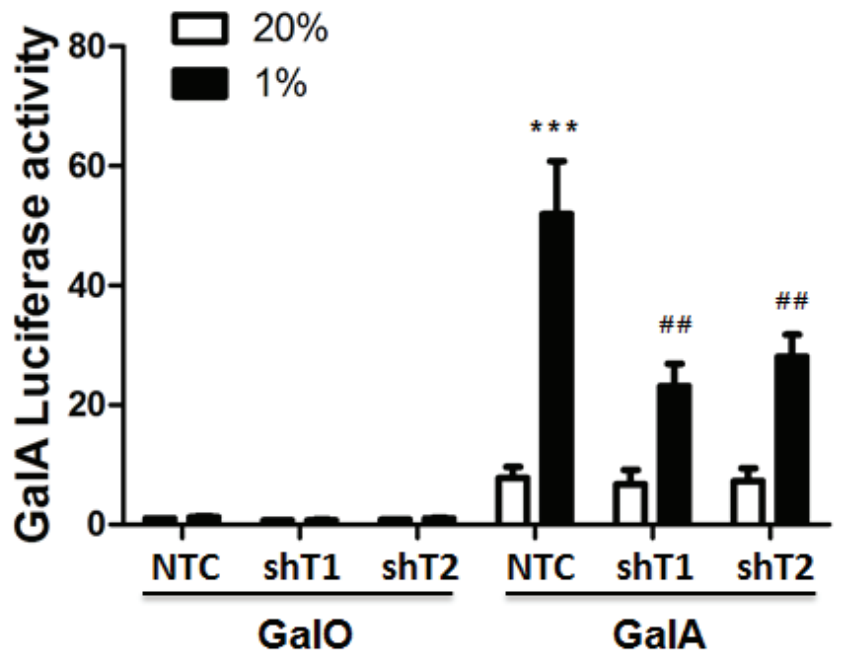

GST-HIF-1a

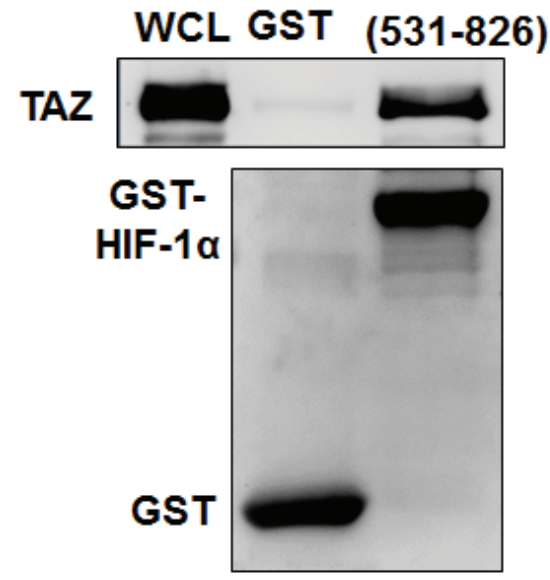

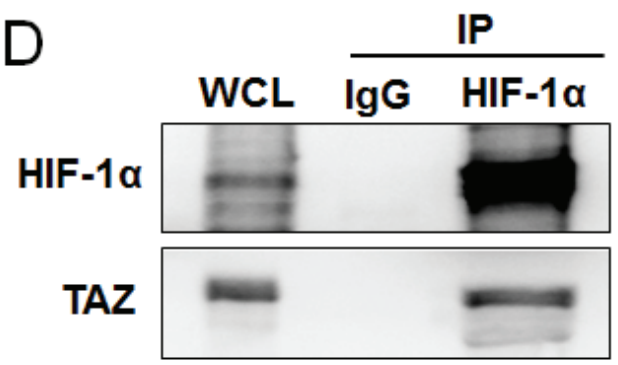

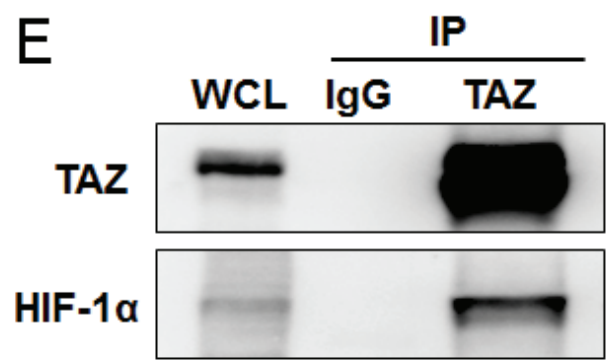

Figure 1: TAZ serves a co-activator for HIF-1 $а$. A. MDA-MB-231 cells were stably transduced with a lentiviral vector encoding shRNA targeting TAZ (shT1 or shT2) or a non-targeting control shRNA (NTC). These subclones were transiently co-transfected with HIF-1-dependent firefly luciferase reporter plasmid p2.1 and control Renilla luciferase reporter plasmid pSV-Renilla, and exposed to 20\% or $1 \% \mathrm{O}_{2}$ for $24 \mathrm{~h}$. The ratio of firefly:Renilla luciferase activity was normalized to lane 1 (mean $\pm \mathrm{SEM}, n=4$ ). ${ }^{* * *} P<0.001$ versus NTC at $20 \% \mathrm{O}_{2} ;{ }^{\prime} P<0.001$ versus NTC at $1 \% \mathrm{O}_{2}$. B. MDA-MB-231 subclones were transiently co-transfected with expression vector pGalO (encoding GAL4 DNA-binding domain) or pGalA (encoding GAL4 DNA-binding domain fused to amino acid residues 531-826 of HIF$1 \alpha$ ), GAL4-dependent firefly-luciferase reporter-plasmid pG5E1bLuc, and pSV-Renilla. Transfected cells were exposed to $20 \%$ or $1 \% \mathrm{O}_{2}$ for $24 \mathrm{~h}$. The luciferase activity was normalized to lane 1 (mean $\pm \mathrm{SEM}, n=4$ ). ${ }^{* * *} P<0.001$ versus NTC at $20 \% \mathrm{O}_{2}$; $P<0.01$ versus NTC at $1 \% \mathrm{O}_{2}$. C. GST pull-down assays were performed with GST or a GST fusion protein containing residues 531-826 of HIF-1 $\alpha$ and whole cell lysate (WCL) from MDA-MB-231 cells that were exposed to $1 \% \mathrm{O}_{2}$ for $24 \mathrm{~h}$. Immunoblot assays were performed with antibodies against TAZ (upper panel) and GST (lower panel). D-E. Immunoprecipitation was performed with anti-HIF-1 $\alpha$ (D), anti-TAZ (E), or IgG (D and E) antibodies and WCL from MDA-MB-231 cells that were exposed to $1 \% \mathrm{O}_{2}$ for $4 \mathrm{~h}$. The immunoprecipitates were analyzed by immunoblot assays using antibodies against HIF-1 $\alpha$ and TAZ. 
HIF-1 $\alpha$ and TAZ [5] as well as HIF-1 $\beta$ (Figure 2A) protein expression. A 247-bp CTGF proximal promoter sequence, which contains three copies of the TEAD-binding site sequence (5'-GGAATG-3') and no match to the HIF-1 binding site consensus sequence (5'-RCGTG-3'), was inserted into the promoterless firefly luciferase reporter plasmid pGL2-Basic to generate pGL2-CTGF. MCF-7 subclones were transiently transfected with pSV-Renilla and either pGL2-CTGF or pGL2-Basic. Luciferase activity was increased in response to hypoxia in NTC cells transfected with pGL2-CTGF, whereas knockdown of HIF-1 $\alpha$, HIF-1 $\beta$, or TAZ significantly decreased luciferase activity under hypoxia and the effect of HIF$1 \alpha$ knockdown was greater than the effect of HIF-1 $\beta$ knockdown (Figure 2A).

The loss of $C T G F$ promoter reporter activity in hypoxic cells with HIF-1 $\alpha$ or HIF- $1 \beta$ knockdown reflected the loss of HIF-1-mediated TAZ mRNA and protein expression [5]. However, the greater inhibitory effect of HIF-1 $\alpha$ knockdown suggested that HIF-1 $\alpha$ might also directly stimulate TAZ transcriptional activity through its physical interaction with TAZ. To test this hypothesis, we expressed a constitutively active form of HIF-1 $\alpha$ (with mutation of the two proline residues that are subject to $\mathrm{O}_{2}-$ dependent hydroxylation) under non-hypoxic conditions in HIF-1 $\beta$ knockdown MCF-7 cells to eliminate HIF1-dependent activation of WWTR1 gene transcription. Under these conditions, $C T G F$ promoter activity was still increased by HIF-1 $\alpha$ and this effect was lost when TAZ expression was knocked down (Figure 2B), indicating that HIF-1 $\alpha$ functions as a co-activator of TAZ-dependent transcription.
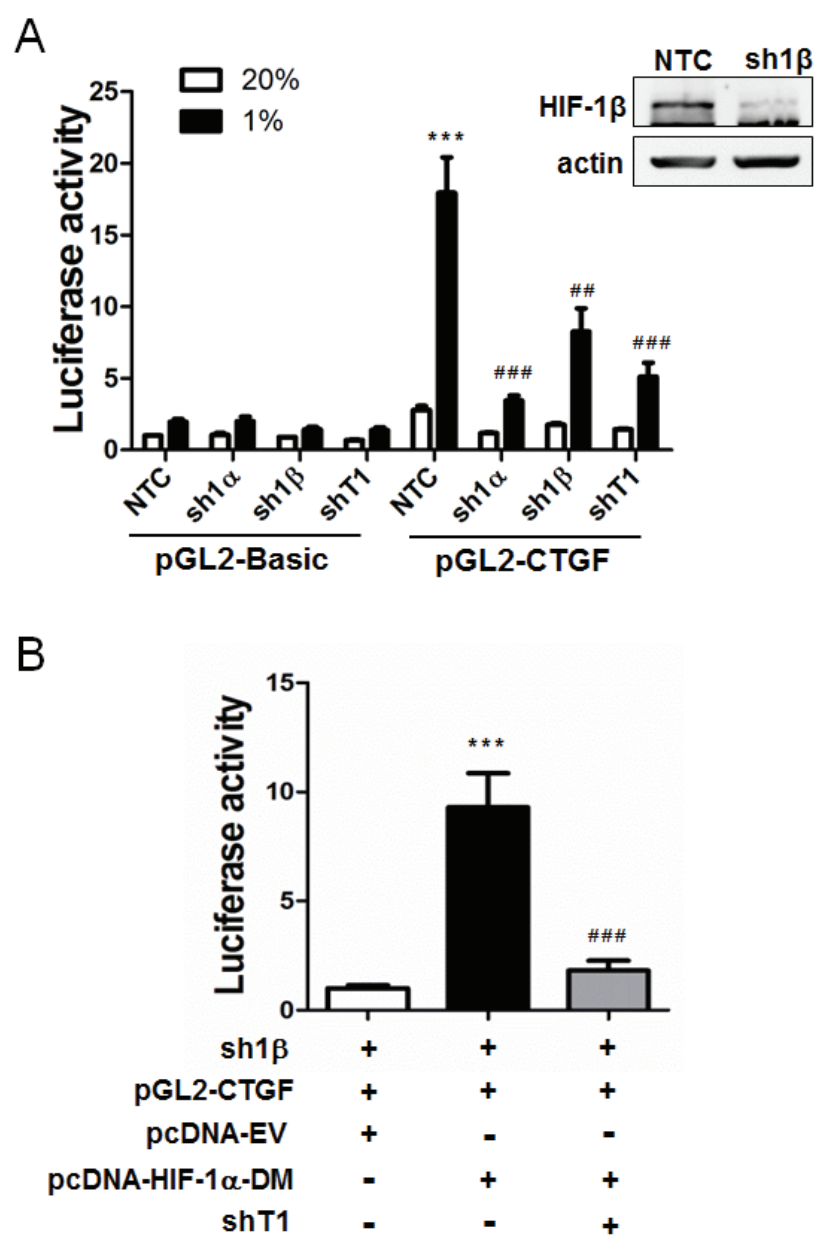

Figure 2: HIF-1 a serves as a co-activator for TAZ. A. MCF-7 cells were stably transfected with a lentiviral expression vector encoding a non-targeting control shRNA (NTC) or shRNA targeting HIF-1 $\alpha(\operatorname{sh} 1 \alpha)$, HIF-1 $\beta$ (sh1 $\beta$; efficiency of knockdown shown in immunoblot at upper right) or TAZ (shT1). The MCF-7 subclones were transiently transfected with pSV-Renilla and either the pGL2-Basic or pGL2-CTGF firefly luciferase reporter, and exposed to $20 \%$ or $1 \% \mathrm{O}_{2}$ for $24 \mathrm{~h}$. The luciferase activity was normalized to lane 1 (mean \pm SEM, n = 4). ${ }^{* * *} P<0.001$ versus NTC at $20 \% \mathrm{O}_{2} ;{ }^{\#} P<0.01,{ }^{\# \#} P<0.001$ versus NTC at $1 \% \mathrm{O}_{2}$. B. MCF-7 cells stably transfected with HIF-1 $\beta$ shRNA $(\operatorname{sh} 1 \beta)$ vector were transiently transfected with pGL2-CTGF, pSV-Renilla, and either pcDNA-EV (empty vector) or pcDNA-HIF-1 $\alpha$-DM (encoding double mutant HIF-1 $\alpha$ [P402A/P564A]) in the presence of TAZ shRNA (shT1) or NTC vector. The luciferase activity was normalized to lane 1 (mean $\pm \mathrm{SEM}, n=4)$ ) ${ }^{* * *} P<0.001$ versus lane 1 ; ${ }^{\# \# \#} P<0.001$ versus lane 2 . 


\section{Co-activator functions of TAZ and HIF-1a contribute to the transcription of endogenous target genes}

We next investigated whether TAZ and HIF-1 $\alpha$ function as reciprocal co-activators in transcription of their endogenous target genes. We reported previously that CTGF mRNA expression increased in MCF-7 cells in response to hypoxia, but induction was lost when expression of HIF-1 $\alpha$ or TAZ was silenced by shRNA [5]. To investigate whether TAZ recruits HIF- $1 \alpha$ and HIF-1 $\beta$ to the $C T G F$ promoter, chromatin immunoprecipitation (ChIP) assays were performed in MCF-7 cells stably transduced with NTC or shT1 expression vector. ChIP primers were designed to amplify the region of the $C T G F$ proximal promoter, which was analyzed by reporter assays above, containing three TEAD binding sites and no HIF-1 binding sites (Figure 3A). Prior studies demonstrated that TAZ-dependent transcriptional co-activation of the $C T G F$ promoter was TEAD-dependent $[14,15]$. Hypoxia induced the binding of HIF-1 $\alpha$ (Figure 3B), but not HIF-1 $\beta$ (Figure $3 \mathrm{C}$ ), to the $C T G F$ promoter in NTC cells, but not in TAZ knockdown cells, demonstrating that TAZ recruits HIF-1 $\alpha$ to the $C T G F$ proximal promoter in hypoxic breast cancer cells.

To determine whether TAZ regulates the expression of HIF-1 target genes, MDA-MB-231 cells, which were stably transduced with NTC or shT1 vector, were exposed to $20 \%$ or $1 \% \mathrm{O}_{2}$ for $24 \mathrm{~h}$ and total RNA was isolated. TAZ knockdown significantly decreased the hypoxiainduced expression of the HIF-1 target genes PDKI
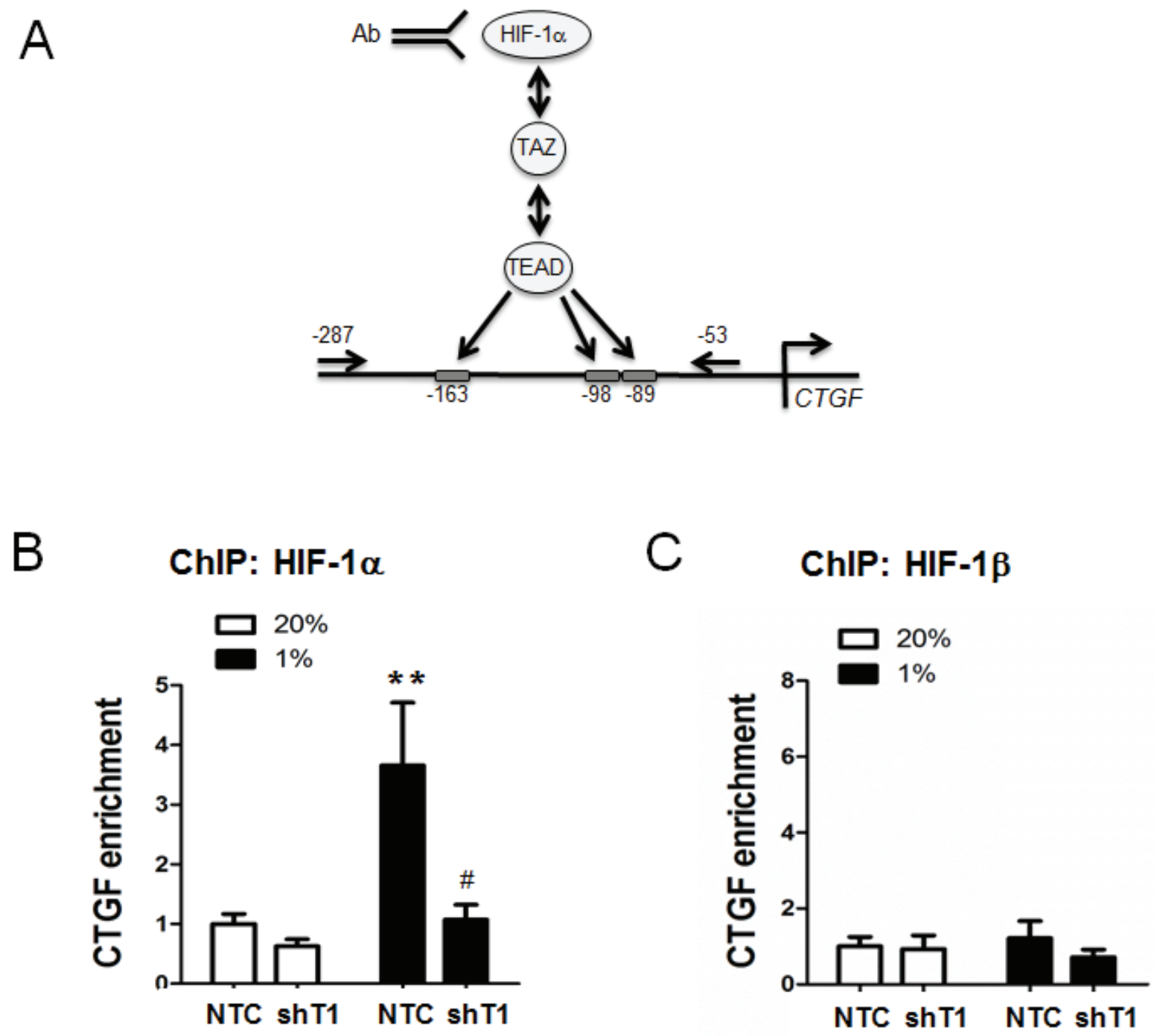

Figure 3: Chromatin immunoprecipitation (ChIP) assays of selective HIF-1 $\alpha$ binding to the CTGF proximal promoter. A. Schematic diagram of primers specific for the $C T G F$ proximal promoter, which contains 3 TEAD binding sites and no HIF-1 binding sites. B-C. MCF-7 subclones (NTC and shT1) were exposed to $20 \%$ or $1 \% \mathrm{O}_{2}$ for $16 \mathrm{~h}$. Chromatin was immunoprecipitated with anti-HIF$1 \alpha(\mathbf{B})$ or anti-HIF-1 $\beta(\mathbf{C})$ antibody and analyzed by qPCR with ChIP primers shown in panel A (mean $\pm \mathrm{SEM}, n=3) .{ }^{* *} P<0.01$ versus NTC at $20 \% ; " P<0.05$ versus NTC at $1 \% \mathrm{O}_{2}$. 
(Figure 4A), LDHA (Figure 4B), BNIP3 (Figure 4C) and P4HA2 (Figure 4D), whereas TAZ knockdown had no significant effect on the expression of these genes in non-hypoxic cells (Figure 4A-4D). ChIP assays revealed hypoxia-induced binding of TAZ to the HRE located in the 5 '-flanking region of the $P D K 1$ gene, which was abrogated by knockdown of HIF-1 $\alpha$ or HIF-1 $\beta$ (Figure $5 \mathrm{~A}$ ). In addition, hypoxia-induced binding of HIF- $1 \alpha$ (Figure $5 \mathrm{~B}$ ) and HIF-1 $\beta$ (Figure 5C) to the HRE in the PDK1 gene was significantly decreased in TAZ knockdown cells. Taken together, the data presented in Figures 3 to 5 demonstrate that HIF- $1 \alpha$ functions as a co-activator for the transcription of TAZ/TEAD target genes and TAZ functions as a co-activator for the transcription of HIF-1 target genes in hypoxic human breast cancer cells.

\section{DISCUSSION}

HIF-1 $\alpha$ plays critical roles in tumor angiogenesis and metabolic reprogramming, as well as multiple steps in the process of breast cancer invasion and metastasis $[3,23]$. HIF-1 and TAZ/TEAD transcriptional activity appear to play important roles in driving breast cancer progression, particularly maintenance of the breast cancer stem cell phenotype $[5,16]$. In a previous study, we demonstrated that under hypoxic conditions, HIF1 increased transcription of the WWTR 1 gene encoding TAZ and the SIAH1 gene encoding a ubiquitin ligase that triggers degradation of LATS2, a negative regulator of TAZ nuclear localization in breast cancer cells [5]. HIF-1 was also reported to transactivate the SIAH2 gene to degrade LATS2 and stabilize YAP, which is a TAZ homolog that also serves as a co-activator for TEAD DNA-binding proteins in the Hippo pathway [24].

B
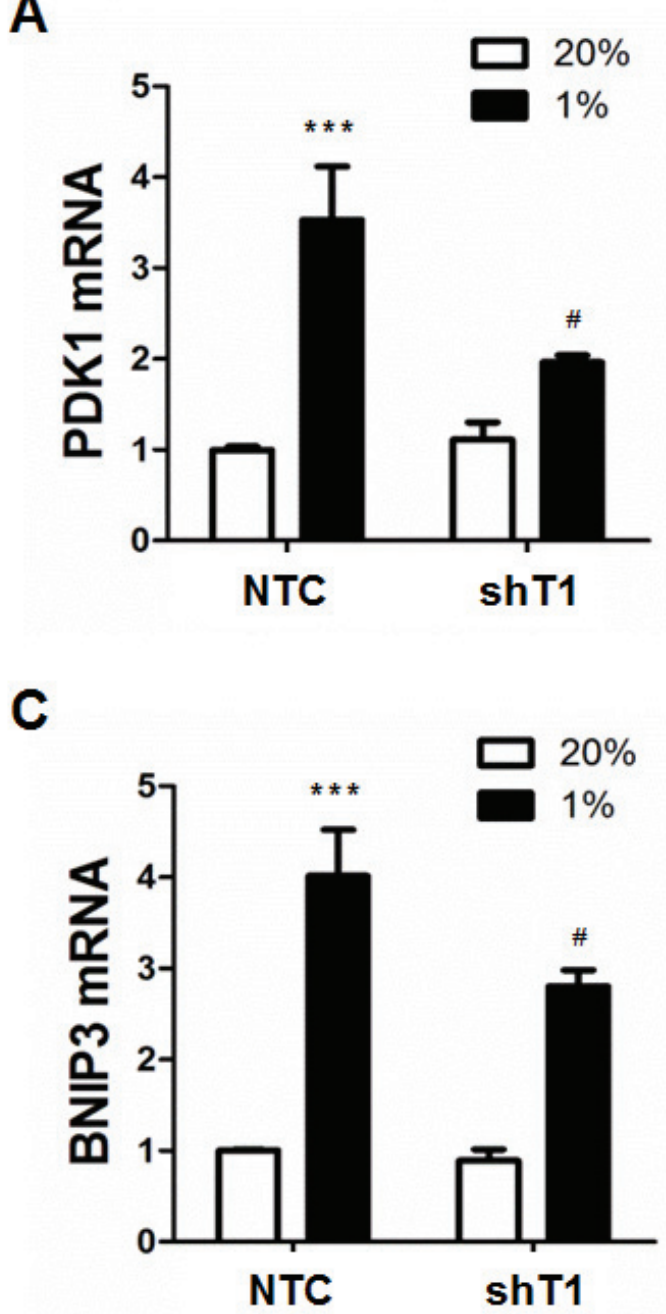
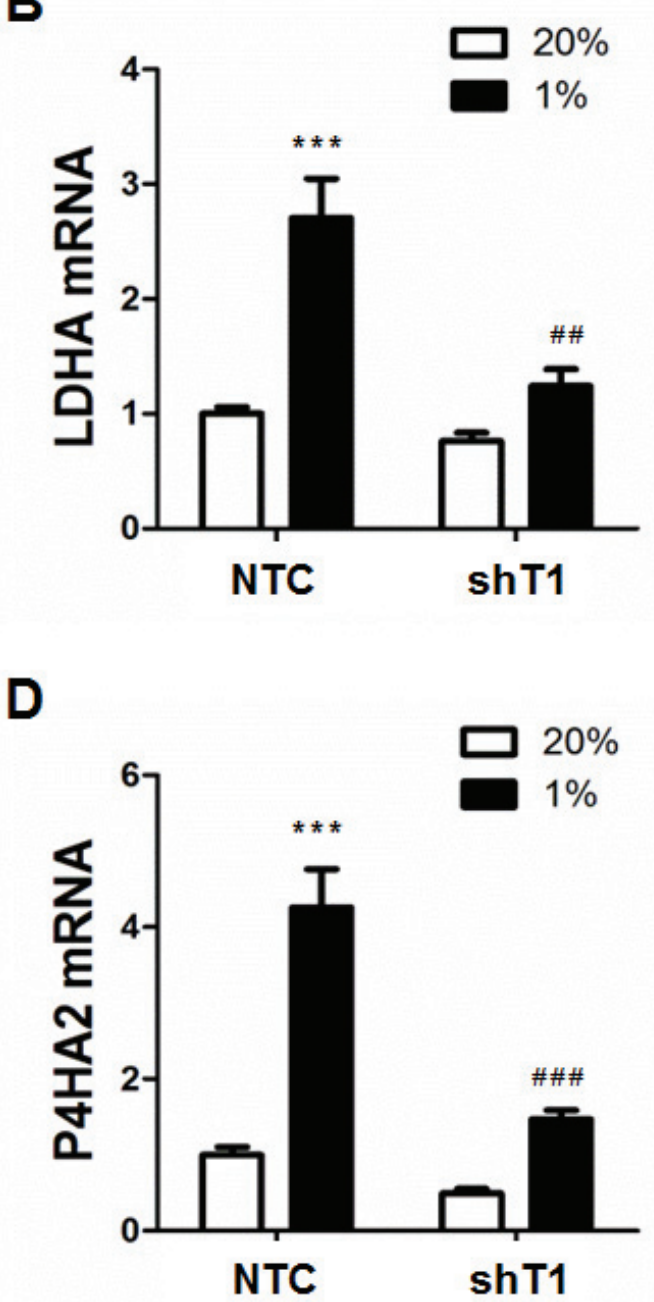

Figure 4: Analysis of HIF-1 target gene expression. A-D. MDA-MB-231 subclones (NTC and shT1) were exposed to 20\% or $1 \% \mathrm{O}_{2}$ for $24 \mathrm{~h}$ and total cellular RNA was isolated. Reverse transcription and qPCR analyses of PDK1 (A), LDHA (B), BNIP3 (C), and P4HA2 (D) mRNAs were performed (mean \pm SEM, $n=3$ ). ${ }^{* * *} P<0.001$ versus NTC at $20 \% \mathrm{O}_{2} ;{ }^{\#} P<0.05,{ }^{\#} P<0.01$, ${ }^{\# \#} P<0.001$ versus $\mathrm{NTC}$ at $1 \% \mathrm{O}_{2}$. 

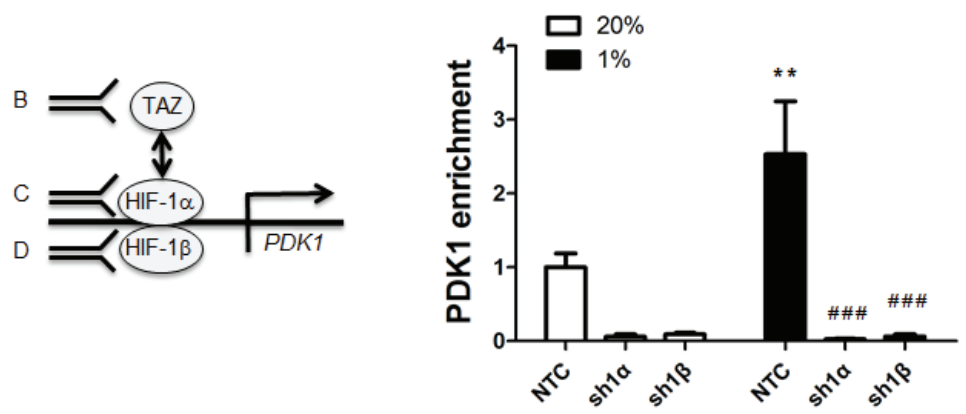

C

ChIP: HIF-1 $\alpha$

D

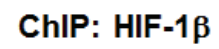
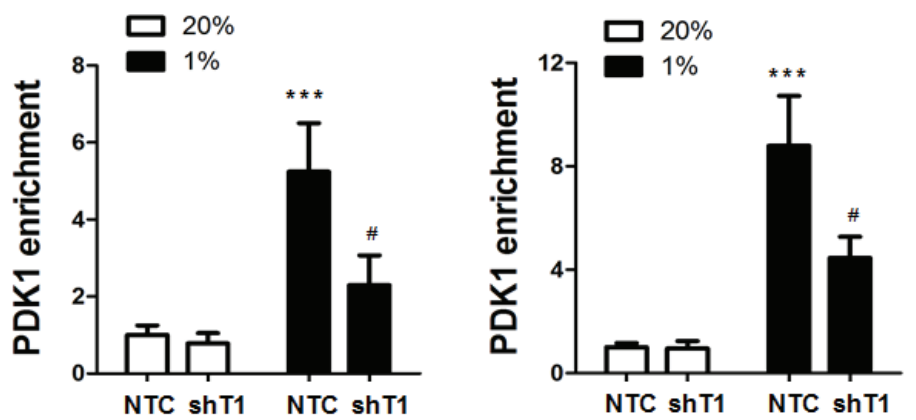

Figure 5: ChIP assays of TAZ and HIF-1 binding to the PDK1 gene in chromatin. A. ChIP assays were performed using antibodies against TAZ (panel B), HIF-1 $\alpha$ (panel C), or HIF-1 $\beta$ (panel D), followed by quantitative real-time PCR with primers spanning the PDK1 hypoxia-response element (mean \pm SEM, $n=3$ ). B. MCF-7 subclones (NTC, sh $1 \alpha$, and sh $1 \beta$ ) were exposed to $20 \%$ or $1 \% \mathrm{O}_{2}$ for $16 \mathrm{~h}$. Chromatin was immunoprecipitated with anti-TAZ antibody. ${ }^{* *} P<0.01$ versus NTC $20 \% \mathrm{O}_{2}$; ${ }^{\ldots \#} P<0.001$ versus NTC at $1 \% \mathrm{O}_{2}$. (C-D) MCF-7 subclones (NTC and shT1) were exposed to $20 \%$ or $1 \% \mathrm{O}_{2}$ for $16 \mathrm{~h}$. Chromatin was immunoprecipitated with anti-HIF-1 $\alpha$ (C) or anti-HIF-1 $\beta$ (D) antibody. ${ }^{* * *} P<0.001$ versus NTC at $20 \% \mathrm{O}_{2} ;{ }^{*} P<0.05$ versus NTC at $1 \% \mathrm{O}_{2}$.

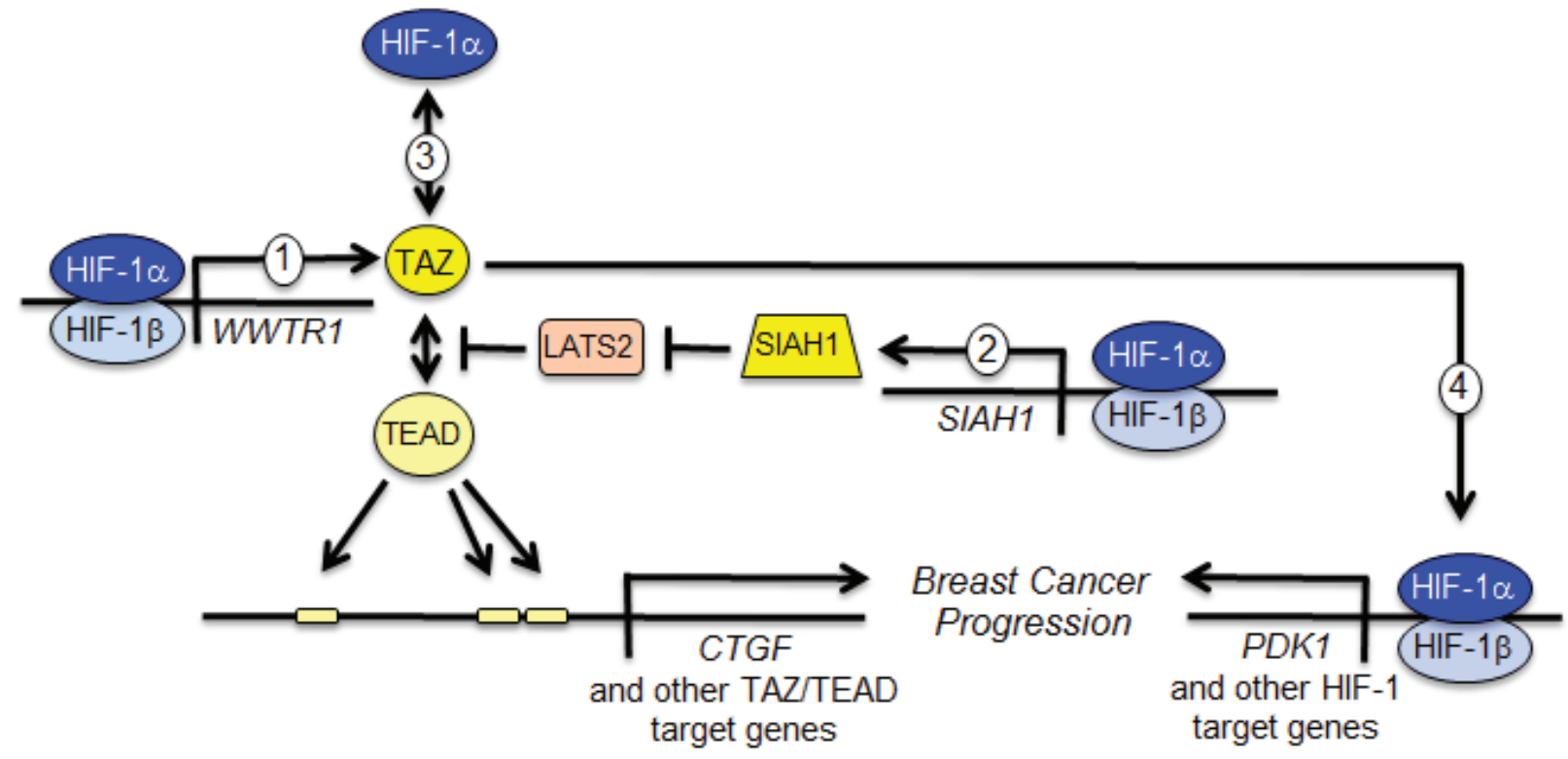

Figure 6: Reciprocal crosstalk promotes breast cancer progression. The schematic summarizes four distinct mechanisms of functional interaction between HIF-1 $\alpha$ and TAZ as determined in this study and previous work [5, 14]. (1) HIF-1 activates transcription of the WWTR1 gene encoding TAZ. (2) HIF-1 activates transcription of the SIAH1 gene encoding an ubiquitin protein ligase that mediates the degradation of LATS2, a negative regulator of TAZ nuclear localization. (3) HIF-1a interacts with TAZ to stimulate transcription of TAZ/ TEAD target genes such as CTGF. (4) TAZ interacts with HIF-1a to stimulate transcription of HIF-1 target genes such as PDK1. 
In the present study, we demonstrate that HIF-1 $\alpha$ physically interacts with TAZ and serves as a co-activator for TEAD/TAZ-dependent transcription, providing a novel molecular mechanism by HIF- $1 \alpha$ stimulates transcription of the $C T G F$ gene, which has been shown to be directly transactivated by either TAZ/TEAD or HIF-1 previously $[5,14,15,25]$. Thus, HIF-1 $\alpha$ may regulate $C T G F$ both as a transcriptional activator (after heterodimerization with HIF-1 $\beta$ and binding to an HRE in the distal promoter) or as a co-activator (by interacting with the TAZ/TEAD complex bound to the proximal promoter). Many other genes are known to be co-regulated by HIF-1 and TAZ, including those encoding plasminogen activator inhibitor 1 and the anti-apoptotic factor survivin [5]. Further studies are required to determine whether HIF-1 also regulates those genes both directly, as a transcriptional activator, and indirectly as a TAZ/TEAD co-activator.

HIF- $1 \alpha$ and TAZ may also complement each other by activating different genes that function in the same pathway. For example, both HIF- $1 \alpha$ and TAZ play key roles in remodeling of the extracellular matrix, which is critical for tissue invasion [26, 27]. We previously demonstrated that HIF- $1 \alpha$ and TAZ cooperate to induce the breast cancer stem cell phenotype [5] but further studies are required to determine the specific target genes and mechanisms of transcriptional activation and coactivation that mediate this effect.

We also show that the TAZ-HIF-1 $\alpha$ interaction stimulates HIF-1 target gene expression, such that TAZ and HIF-1 $\alpha$ function as reciprocal co-activators. A previous study reported that TAZ served as a HIF-1 co-activator in breast cancer cells selected for metastasis to bone [18], whereas our study expands this co-activator function to MDA-MB-231 breast cancer cells, which metastasize to lungs and lymph nodes, as well as non-metastatic MCF7 cells. We demonstrate that one of the mechanisms by which TAZ stimulates HIF-1 transcriptional activity is by stabilizing its binding to hypoxia response elements (Figure 5C-5D), which has been observed for other HIF-1 co-activators, including JMJD2C and PKM2 [10, 11].

Taken together with our previous study [5], we have now identified four discrete mechanisms of crosstalk between HIF-1 $\alpha$ and TAZ that serve to increase the activity of both pathways (Figure 6) and drive breast cancer progression [5]. Increased expression of both TAZ and HIF-1 target genes in primary breast tumors is associated with increased patient mortality [5], underscoring the clinical consequences of functional interactions between these two transcriptional regulators. Therapeutic strategies that include drugs that inhibit HIF-1 $\alpha$ protein accumulation $[19,20,28]$ or perhaps TAZ target gene products [29] may improve outcome in women with triple negative breast cancer, who are currently treated with cytotoxic chemotherapy with durable response rates of less than $20 \%$.

\section{MATERIALS AND METHODS}

\section{Cell culture}

Breast cancer cell lines MDA-MB-231 and MCF7 were cultured in high-glucose $(4.5 \mathrm{mg} / \mathrm{mL})$ DMEM supplemented with $10 \%(\mathrm{v} / \mathrm{v})$ fetal bovine serum and $1 \%$ (v/v) penicillin-streptomycin (Invitrogen). All cells were maintained at $37^{\circ} \mathrm{C}$ in a $5 \% \mathrm{CO}_{2}, 95 \%(\mathrm{v} / \mathrm{v})$ air incubator. For hypoxic exposure, cells were placed in a modular incubator chamber (Billups-Rothenberg) and flushed with a $1 \% \mathrm{O}_{2} / 5 \% \mathrm{CO}_{2} / 94 \% \mathrm{~N}_{2}(\mathrm{v} / \mathrm{v})$ gas mixture.

\section{ShRNA, lentiviruses, and transduction}

Vectors encoding shRNA targeting HIF-1 $\alpha$ and TAZ were previously described [5]. The sense strand of the 21-nucleotide sequence encoding shRNA targeting HIF-1 $\beta$ was 5'-GCCTACACTCTCCAACACAAT-3'. Oligonucleotides were annealed and ligated into $\mathrm{AgeI} /$ EcoRI-linearized pLKO.1-puro lentiviral vector. The lentiviral vectors were co-transfected with plasmid pCMVdR8.91 and a plasmid encoding vesicular stomatitis virus $\mathrm{G}$ protein into 293-T cells using Lipofectamine 2000 (Invitrogen). Medium containing viral particles was collected $48 \mathrm{~h}$ after transfection and passed through a $0.45-\mu \mathrm{M}$ filter. MDA-MB-231 and MCF-7 cells were transduced with viral supernatant supplemented with 8 $\mu \mathrm{g} / \mathrm{mL}$ Polybrene (Sigma-Aldrich). After $24 \mathrm{~h}$, cells were selected in medium containing $0.6 \mu \mathrm{g} / \mathrm{mL}$ puromycin (Sigma-Aldrich).

\section{Luciferase reporter plasmid constructs}

A 247-bp CTGF proximal promoter sequence was amplified from human genomic DNA by PCR (primers: 5'-CCCCTCGAGAGTGTGCCAGCTTTTTCAGAC-3' and 5'-CGAAGCTTCGAGCTGGAGGGTGGAGT-3'), purified by gel extraction, and inserted into the XhoI and HindIII sites of pGL2-Basic (Promega). Plasmid constructs were confirmed by nucleotide sequencing.

\section{Reverse transcription and real-time quantitative PCR (qPCR)}

Total cellular RNA was extracted using TRIzol (Invitrogen), precipitated with isopropanol, treated with DNase I (Ambion), and reverse transcribed using the iScript cDNA Synthesis kit (Bio-Rad). qPCR analysis was performed using Maxima SYBR Green Master Mix (Fermentas) with the iCycler Real-time PCR Detection System (BioRad). The $2^{-\Delta \Delta \mathrm{Ct}}$ method was used to calculate relative gene expression [19]. Results were normalized to 
the 18S rRNA signal. Primer sequences are as follows:

PDK1, 5'-GGATTGCCCATATCACGTCTTT-3'

and 5'-TCCCGTAACCCTCTAGGGAATA-3';

LDHA, 5'-ATCTTGACCTACGTGGCTTGGA-3'

and 5'-CCATACAGGCACACTGGAATCTC-3';

BNIP3, 5'-CAGGGCTCCTGGGTAGAACT-3'

and 5'-CTACTCCGTCCAGACTCATGC-3';

P4HA2, 5'-GCCTGCGCTGGAGGACCTTG-3'

and 5'-TGTGCCTGGGTCCAGCCTGT-3'; $18 \mathrm{~S}$

rRNA, 5'-GAGGATGAGGTGGAACGTGT-3' and

5'-AGAAGTGACGCAGCCCTCTA-3'.

\section{Immunoprecipitation and immunoblot assays}

Whole cell lysates were prepared in RIPA lysis buffer. $30 \mu \mathrm{l}$ of protein G-Sepharose beads (GE Healthcare) and immunoprecipitating antibody were incubated with $0.75 \mathrm{mg}$ of cell lysate overnight at $4^{\circ} \mathrm{C}$. Beads were washed five times in lysis buffer. Proteins were eluted in SDS sample buffer and separated by SDS-PAGE. Antibodies used in immunoprecipitation and immunoblot assays were: HIF-1 $\alpha$ (BD Transduction Laboratory); HIF-1 $\beta$, TAZ and IgG (Novus Biologicals); and GST and $\beta$-actin (Santa Cruz). HRP-conjugated anti-rabbit (Amersham) and anti-mouse (Santa Cruz) secondary antibodies were used. Chemiluminescent signal was developed using ECL Plus (GE Healthcare).

\section{ChIP assay}

ChIP assays were performed as previously described [30]. MCF-7 cells were cross-linked in 3.7\% formaldehyde for $10 \mathrm{~min}$ and lysed with SDS lysis buffer. Chromatin was sheared by sonication and lysates were pre-cleared with salmon sperm DNA/protein A-agarose slurry (Millipore) and incubated with IgG (Novus Biologicals) or antibodies against the following proteins: HIF-1 $\alpha$ (Santa Cruz), HIF$1 \beta$ and TAZ (Novus Biologicals). Salmon sperm DNA/ protein A-agarose slurry was added and the agarose beads were washed sequentially with: low- and high-salt immune complex wash buffers; LiCl immune complex wash buffer; and twice with TE buffer. DNA was eluted in 1\% SDS with $0.1 \mathrm{M} \mathrm{NaHCO} 3$, and crosslinks were reversed by addition of $0.2 \mathrm{M} \mathrm{NaCl}$. DNA was purified by phenolchloroform extraction and ethanol precipitation, suspended in $50 \mu \mathrm{l}$ TE buffer, and a $2-\mu 1$ aliquot was used for qPCR. The nucleotide sequences of primers used for qPCR are as follows: CTGF, 5'-GGAGTGGTGCGAAGAGGATA-3' and 5'-GCCAATGAGCTGAATGGAGT-3'; and PDK1, 5'-CGCGTTTGGATTCCGTG-3' and 5'-CCAGTTATAATCTGCCTTCCCTATTATC-3'.

\section{GST pull-down assay}

GST fusion proteins were expressed in Escherichia coli BL21-Gold (DE3) and purified as described [10, 11, 31]. For GST pull-down from cell lysates, equal molar amounts of GST and GST fusion proteins immobilized on glutathione-Sepharose 4B beads were incubated overnight with $2 \mathrm{mg}$ of whole cell lysates. After washing five times, the bound proteins were fractionated by SDS-PAGE, followed by immunoblot assays.

\section{Luciferase reporter assays}

Cells were seeded onto 48-well plates. For p2.1 assays, cells were co-transfected with p2.1 [21] and pSVRenilla. For GalA assays, cells were co-transfected with pG5E1bLuc and either pGalA or pGalO [22]. For CTGF promoter assays, cells were co-transfected with pSVRenilla, either pGL2-CTGF or pGL2-Basic, and either pcDNA3.1 empty vector or pcDNA3.1-HIF- $1 \alpha(\mathrm{P} 402 \mathrm{~A} /$ P564A) [10]. Transfected cells were exposed to $20 \%$ or $1 \% \mathrm{O}_{2}$ for $24 \mathrm{~h}$. Firefly and Renilla luciferase activities in cell lysates were determined using the Dual-Luciferase Assay System (Promega).

\section{Statistical analysis}

Data are presented as mean \pm SEM and were analyzed with an unpaired two-tailed Student's $t$ test for two groups or ANOVA followed by Bonferroni post-test for multiple groups. $P<0.05$ was considered significant.

\section{ACKNOWLEDGMENTS}

The authors are grateful to Karen Padgett of Novus Biologicals for generously providing IgG and antibodies against HIF-1 $\beta$ and TAZ. L.X. was supported by grants from the China Scholarship Council and the National Natural Science Foundation of China (NSFC No. 81402526). D.M.G. and W.L. were supported by National Cancer Institute grants K99-CA181352 and K99-CA168746, respectively. G.L.S. is an American Cancer Society Research Professor and the C. Michael Armstrong Professor at the Johns Hopkins University School of Medicine.

\section{CONFLICTS TO INTEREST}

There are no conflicts to declare.

\section{REFERENCES}

1. Vaupel P, Mayer A and Höckel M. Tumor hypoxia and malignant progression. Methods Enzymol 2004;381:335- 
354.

2. Harris AL. Hypoxia-a key regulatory factor in tumor growth. Nat Rev Cancer 2002;2:38-47.

3. Semenza GL. Hypoxia-inducible factors: mediators of cancer progression and targets for cancer therapy. Trends Pharmacol Sci 2012;33:207-214.

4. Conley SJ, Gheordunescu E, Kakarala P, Newman B, Korkaya H, Heath AN, Clouthier SG, Wicha MS. Antiangiogenic agents increase breast cancer stem cells via the generation of tumor hypoxia. Proc Natl Acad Sci U S A 2012;109:2784-2789.

5. Xiang L, Gilkes DM, Hu H, Takano N, Luo W, Lu H, Bullen JW, Samanta D, Liang H and Semenza GL. Hypoxia-inducible factor 1 mediates TAZ expression and nuclear localization to induce the breast cancer stem cell phenotype. Oncotarget 2014; 5:12509-12527.

6. Samanta D, Gilkes DM, Chaturvedi P, Xiang L, Semenza GL. Hypoxia-inducible factors are required for chemotherapy resistance of breast cancer stem cells. Proc Natl Acad Sci U S A 2014;111:E5429-E5438.

7. Wang GL, Jiang BH, Rue EA and Semenza GL. Hypoxiainducible factor 1 is a basic- helix-loop-helix-PAS heterodimer regulated by cellular O2 tension. Proc Natl Acad Sci U S A 1995;92:5510-5514.

8. Prabhakar NR and Semenza GL. Adaptive and maladaptive cardiorespiratory responses to continuous and intermittent hypoxia mediated by hypoxia-inducible factors 1 and 2 . Physiol Rev 2012;92:967-1003.

9. Arany Z, Huang LE, Eckner R, Bhattacharya S, Jiang C, Goldberg MA, Bunn HF and Livingston DM. An essential role for $\mathrm{p} 300 / \mathrm{CBP}$ in the cellular response to hypoxia. Proc Natl Acad Sci U S A 1996;93:12969-12973.

10. Luo W, Hu H, Chang R, Zhong J, Knabel M, O’Meally R, Cole RN, Pandey A and Semenza GL. Pyruvate kinase M2 is a PHD3-stimulated coactivator for hypoxia- inducible factor 1. Cell 2011;145:732-744.

11. Luo W, Chang R, Zhong J, Pandey A and Semenza GL. Histone demethylase JMJD2C is a coactivator for hypoxia-inducible factor 1 that is required for breast cancer progression. Proc Natl Acad Sci U S A 2012;109:E3367-E3376.

12. Lee JS, Kim Y, Bhin J, Shin HJ, Nam HJ, Lee SH, Yoon JB, Binda O, Gozani O, Hwang D, Baek SH. Hypoxia-induced methylation of a pontin chromatin remodeling factor. Proc Natl Acad Sci U S A 2011;108:13510-13515.

13. Chen X, Iliopoulos D, Zhang Q, Tang Q, Greenblatt MB, Hatziapostolou M, Lim E, Tam WL, Ni M, Chen Y, Mai J, Shen H, Hu DZ, et al. XBP1 promotes triple-negative breast cancer by controlling the HIF-1 $\alpha$ pathway. Nature 2014;508:103-107.

14. Zhang H, Liu CY, Zha ZY, Zhao B, Yao J, Zhao S, Xiong Y, Lei QY and Guan KL. TEAD transcription factors mediate the function of TAZ in cell growth and epithelialmesenchymal transition. J Biol Chem 2009;284:13355-
13362.

15. Lai D, Ho KC, Hao $\mathrm{Y}$ and Yang $\mathrm{X}$. Taxol resistance in breast cancer cells is mediated by the hippo pathway component TAZ and its downstream transcriptional targets Cyr61 and CTGF. Cancer Res 2011;71:2728-2738.

16. Cordenonsi M, Zanconato F, Azzolin L, Forcato M, Rosato A, Frasson C, Inui M, Montagner M, Parenti AR, Poletti A, Daidone MG, Dupont S, Basso G, et al. The Hippo transducer TAZ confers cancer stem cell-related traits on breast cancer cells. Cell 2011;147:759-772.

17. Pobbati AV and Hong W. Emerging roles of TEAD transcription factors and its coactivators in cancers. Cancer Biol Ther 2013;14:390-398.

18. Bendinelli P, Maroni P, Matteucci E, Luzzati A, Perrucchini $\mathrm{G}$ and Desiderio MA. Hypoxia-inducible factor-1 is activated by transcriptional coactivator with PDZ- binding motif (TAZ) versus WW domain-containing oxidoreductase (WWOX) in hypoxic microenvironment of bone metastasis from breast cancer. Eur J Cancer 2013;49:2608-2618.

19. Zhang H, Wong CC, Wei H, Gilkes DM, Korangath P, Chaturvedi P, Schito L, Chen J, Krishnamachary B, Winnard PT Jr, Raman V, Zhen L, Mitzner WA, et al. HIF1- dependent expression of angiopoietin-like 4 and L1CAM mediates vascular metastasis of hypoxic breast cancer cells to the lungs. Oncogene 2012;31:1757- 1770.

20. Schito L, Rey S, Tafani M, Zhang H, Wong CC, Russo A, Russo MA and Semenza GL. Hypoxia-inducible factor 1-dependent expression of platelet-derived growth factor B promotes lymphatic metastasis of hypoxic breast cancer cells. Proc Natl Acad Sci U S A 2012;109:E2707-E2716.

21. Semenza GL, Jiang BH, Leung SW, Passantino R, Concordet JP, Maire $\mathrm{P}$ and Giallongo A. Hypoxia response elements in the aldolase $\mathrm{A}$, enolase 1 , and lactate dehydrogenase A gene promoters contain essential binding sites for hypoxia-inducible factor 1. J Biol Chem 1996;271:32529-32537.

22. Jiang BH, Zheng JZ, Leung SW, Roe R and Semenza GL. Transactivation and inhibitory domains of hypoxiainducible factor $1 \alpha$. Modulation of transcriptional activity by oxygen tension. J Biol Chem 1997;272:19253-19260.

23. Semenza GL. Oxygen sensing, hypoxia-inducible factors, and disease pathophysiology. Annu Rev Pathol 2014;9:4771.

24. Ma B, Chen Y, Chen L, Cheng H, Mu C, Li J, Gao R, Zhou C, Cao L, Liu J, Zhu Y, Chen Q and Wu S. Hypoxia regulates Hippo signaling through the SIAH2 ubiquitin E3 ligase. Nat Cell Biol 2015;17:95-103.

25. Higgins DF, Biju MP, Akai Y, Wutz A, Johnson RS and Haase VH. Hypoxic induction of Ctgf is directly mediated by HIF-1. Am J Physiol Renal Physiol 2004;287:F1223F1232.

26. Chang C, Goel H, Pursell B, Shultz LD, Greiner DL, Ingerpuu S, Patarroyo M, Cao S, Lim E, Mao J, McKee KK, Yurchenco PD and Mercurio AM. A laminin 511 
matrix is regulated by TAZ and functions as the ligand for the $\alpha 6 \beta 1$ integrin to sustain breast cancer stem cells. Genes Dev 2015;29:1-6.

27. Gilkes DM, Semenza GL, Wirtz D. Hypoxia and the extracellular matrix: drivers of tumor metastasis. Nat Rev Cancer 2014;14:430-439.

28. Xiang L, Gilkes DM, Chaturvedi P, Luo W, Hu H, Takano N, Liang H and Semenza GL. Ganetespib blocks HIF-1 activity and inhibits tumor growth, vascularization, stem cell maintenance, invasion, and metastasis in orthotopic mouse models of triple- negative breast cancer. J Mol Med (Berl) 2014;92:151-64.

29. Frangou C, Li YW, Shen H, Yang N, Wilson KE, Blijlevens M, Guo J, Nowak NJ and Zhang J. Molecular profiling and computational network analysis of TAZ-mediated mammary tumorigenesis identifies actionable therapeutic targets. Oncotarget 2014;5:12166-12176.

30. Chaturvedi P, Gilkes DM, Wong CC, Luo W, Zhang H, Wei H, Takano N, Schito L, Levchenko A and Semenza GL. Hypoxia-inducible factor-dependent breast cancermesenchymal stem cell bidirectional signaling promotes metastasis. J Clin Invest 2013;123:189-205.

31. Baek JH, Liu YV, McDonald KR, Wesley JB, Hubbi ME, Byun H and Semenza GL. Spermidine/spermineN1-acetyltransferase 2 is an essential component of the ubiquitin ligase complex that regulates hypoxia-inducible factor 1 $\alpha$. J Biol Chem 2007; 282:23572-23580. 